\title{
Optimization of Welding Pliers Production for the Automotive Industry - Case Study
}

\author{
Nikoleta Mikušová' ${ }^{*}$, Soňa Badiarová1, Karel Jeřábek² \\ 1 Technical University of Kosice, Faculty of Mining, Ecology, Process Control and Geotechnologies, Institute of \\ Logistics and Transport, Letná 9, 04001 Kosice, Slovakia \\ 2 Institute of Technology and Business in České Budějovice, Faculty of Technology, Department of Transport \\ and Logistics, Okružní 10, 37001 České Budějovice, Czech Republic \\ * Corresponding author's e-mail: nikoleta.mikusova@tuke.sk
}

\begin{abstract}
The paper presents a production process optimization by the simulation approach. The research was realized by a case study for an enterprise producing welding pliers for the automotive industry. The solution of the study was realized in the environment of the Tecnomatix Plant Simulation software, which can simulate the production process after choosing the right conditions and connections among the elements. The research aimed at creating a simulation model of the evaluated process with real data and designing optimization solution of the production process, which assumes an increase in the devices and machines utilization. The results showed improvements in the efficiency of the production process, in the particular case increasing by more than $25 \%$.
\end{abstract}

Keywords: simulation, model, optimization, production process.

\section{INTRODUCTION}

The success of a manufacturing enterprise and its ability to compete in its fields requires improving three main aspects: increasing quality, reducing costs and increasing flexibility. Some processes can show signs of inefficiency and it is needed to analyse them as well as - at the same time - find and apply a suitable optimization method. The benefits of lean manufacturing are reduced inventory, less process waste, less rework, reduced lead time, financial savings and increased process understanding [1].

The market environment puts pressure on companies to improve their operating conditions. Production must operate in such a way that the transformation of inputs to outputs takes place at optimal consumption of production inputs, the most appropriate choice of production processes means and with optimal use of production capacity [2]. One of the possibilities for production companies is to apply a simulation approach. The simulation presents the "construction" of a fictitious or real system with its partial processes, which are mediated by the model. Experiment with a simulation model yields the output values that can be further processed and modified and thus it is possible to constantly improve the simulated conditions and apply them in the production processes [3]. However, simulation is not a tool that allows obtaining the optimal solution directly, but rather it is a support tool that helps test the effects of different decisions on the simulation model. The term "model" presents a simplified virtual or real production unit which contains all the sub-processes of the system. It describes the behaviour of elements in the simulation program. The simulation methods differ from other methods in the way of obtaining the solutions that were monitored from the running of the model (program) [4]. This monitoring is often included in a separate simulation model. This model can thus provide results based on the information that is obtained from the changes in the state during the modelled time. 
Simulation can find its application in different areas of research and practice. As it is mentioned in [5], it is possible to use simulation and its principles also in logistics, for example for the planning, evaluation and monitoring of relevant processes. It is possible to say that simulation is a method that allows realizing experiment with the proposed model before the process of it physically operation [6].

Simulation with the help of the simulation model brings a more realistic view of the production processes and therefore many industries try to use this way. For example, it is possible to analyze just-in-time production processes, as it was in the work of Savsar and Al-Jawini. These authors presented a simulation model which was developed to analyze the production system and brings answers to several questions related to the performance of just-in-time systems for production processes [7].

The simulation presents an important component of every production process. It is also necessary for improving the effectiveness of operations in production systems. Chramcov et al. presented an interesting simulation approach to achieving more efficient production systems. They used the simulation by the Witness and also presented a simulation of concrete manufacturing systems [8].

It is possible to apply simulation for production planning support, as it is presented in the work [9]. The authors dealt with the possibilities of using computer simulation during production scheduling. The result of their research study was a simulation model, which after modification and adaptation can be used for the creation of production plans [9].

Another interesting way for simulation application is optimization. In their work, Lim, Alpan and Penz presented a simulation-optimization approach for sales and operations planning with the focus on the automotive industry. They formulated a multiobjective optimization model and its solution by a simulation-optimization approach. Their results and the created model and solution were illustrated, for example with the case study of Renault [10].

Fabianova et al. used quantitative forecasting and computer simulations to create the production plan. These two approaches to production plan creation were demonstrated in a model case study [11].

\section{EVALUATED PRODUCTION PROCESS AND ITS ACTIVITIES}

The research was realized by the help of a case study in the production enterprise. The main activity of the production enterprise is the machining of parts by milling on CNC technological equipment. The research study through simulation will deal with the process of manufacturing a component from a set of welding pliers, which are primarily intended for the automotive industry. The basic scheme of the evaluated process is presented in Figure 1.

The example of the clamping No. 1 (marked as a blue line) is presented of the following sequence:

1. Aligning on a milling machine (duration -2 $\min$ ),

2. Creation of the production program (and 3D model, duration $-30 \mathrm{~min}$ ),

3. Clamping of the part to the Machine_1 (based on the program, the $\mathrm{CNC}$ production is started, duration $-30 \mathrm{~min}$ ),

4. Start of CNC production (duration $-30 \mathrm{~min}$ ),

5. Transfer of the part to the DTC (department of technical control) No.1, an inspection of the part (duration $-2 \mathrm{~min}$ ),

6. Storage of parts in Warehouse No. 2.

A similar procedure is applied also for clamps 2-6. It should be emphasized that the priority of the first clamping (blue line in Fig. 1) is the creation of surfaces, which are then used in the next clamping. At the same time, to shorten the machine time and also to increase the quality, the second clamping is divided into 2 parts. The first part of the semi-finished products is based on the excavation of maximum material (it allows not high-stress pressure, especially for the subsequent clamping No 3. Moreover, the program time of the production process is divided into 2 parts, which will increase the utilization of the machines. On the basis of the detailed study in the operation and production process, the research problem was defined in the requirement of reassessment of the production process, by the help of the simulation model.

The case study was chosen so that the obtained results will be presented in an understandable form and at the same time to show how it is possible to apply the method of computer simulation in real enterprise processes. The method of computer simulation is not only a sterile method detached from reality, but it is a method with a direct application link to industrial practice. By its appropriate application, it is possible to significantly increase the efficiency of enterprise processes at an arbitrary phase of the life cycle of enterprise, production or provided service. 


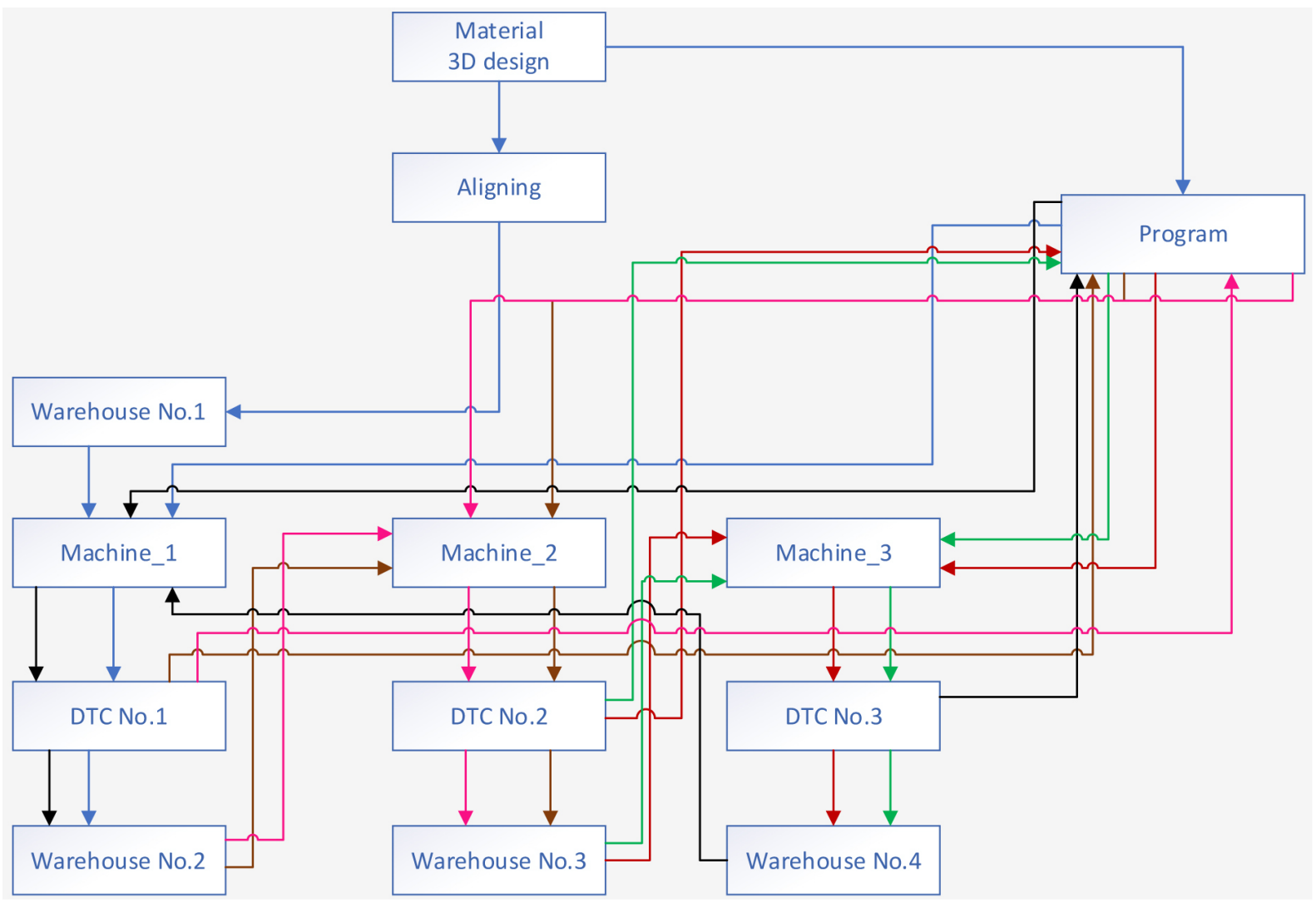

Fig. 1. Scheme of the evaluated production process (Legend - blue line = clamping No. 1 , brown line $=$ clamping No. 2 , red line $=$ clamping No. 3 , black line $=$ clamping No. 4, pink line = clamping No. 5, green line = clamping No. 6)

\section{SIMULATION MODEL}

The Tecnomatix Plant Simulation software was used for the simulation model creation. This software works on the principle of connecting windows with specific properties, with the required definition of relations and conditions for the correct operation of the simulation. These windows can be grouped, so-called Frames. The Frame with the title Input (Figure 2) was created for the closing of input information of the production process. This contains the data about the volume of material in the window Material. Window Drawing presents the drawing documentation and model for customers. This model serves in the simulation as a source for the programmer who creates a CNC program for the machining centre.

Definition of the "Material" window (Figure 3) shows the input number of the material set to 500 pieces, which is the most common volume of the products from the evaluated enterprise by the production process according to the customer's needs.
Workplaces were created by the SingleProc function (activities programming, machine cutting, angling, department of technical control DTC). There is an auxiliary function "Buffer" in the program, which connects individual windows and it helps to enter conditions for the material flow. The "DismantleStation" function divides input source into two parts, where one in the form of material goes to the warehouse. Here it waits for the creation of the $\mathrm{CNC}$ program. The

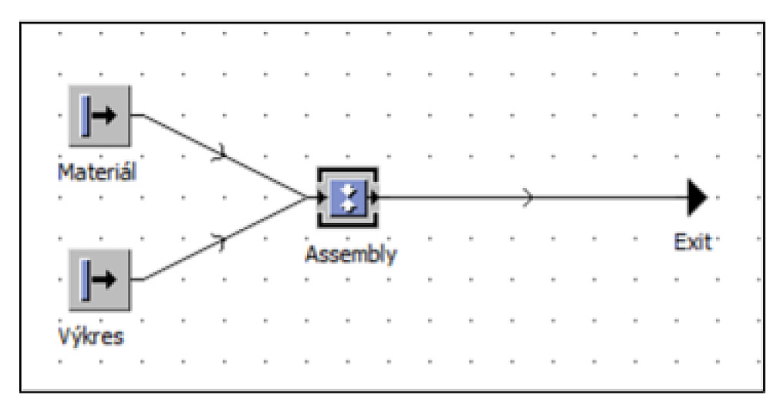

Fig. 2. "Input" Frame - containing program input information 


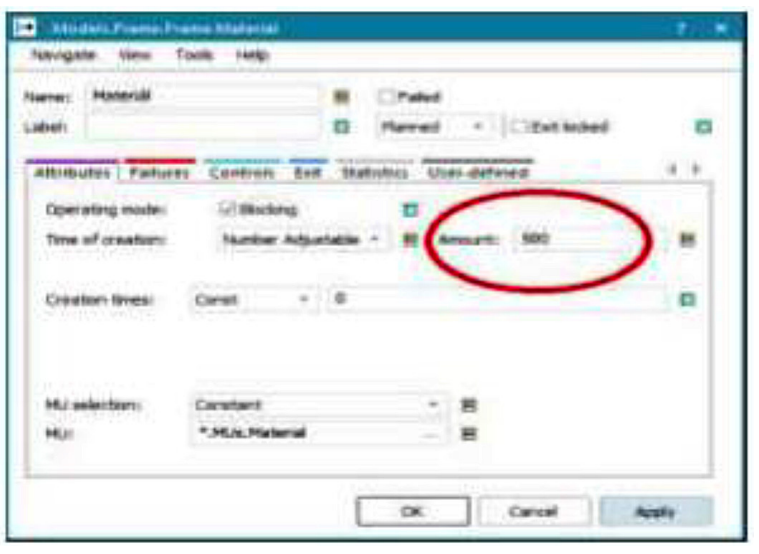

Fig. 3. "Material" Window

Material Flow | Fluids | Resources Information Flow | User Interface | Mobile Units | Tools

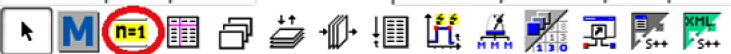

Fig. 4. The "Toolbox" card

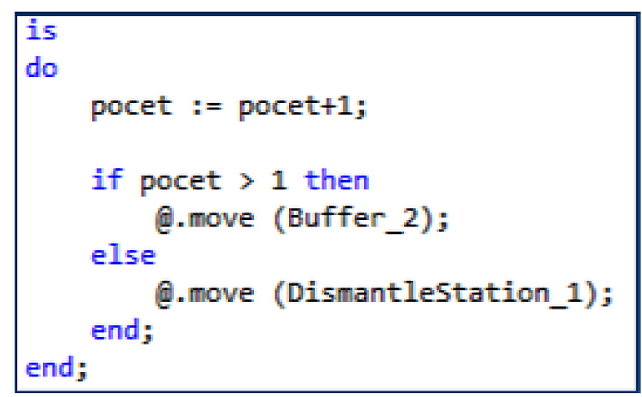

Fig. 5. Condition of the "Buffer_1" window

second part of the source is the input information for the programmer. It contains a drawing and the model of the part. The variables needed to the simulation control were defined using the "Variable" function (Figure 4), which is located on the "Toolbox" card.

The input is connected to the "Buffer_1" function. This has at its output, in the "Controls" card (Figure 6) defined a variable (Figure 5) which takes care of counting the pieces that pass through it as a control element. At the same time, it is an auxiliary variable created for the "if-thenelse" function. This function divides the material according to the number of pieces that passed through "Buffer_1". It the number of pieces in the "Buffer 1" window is greater than 1 , the material goes to "Buffer_2". Otherwise, it goes to the "DismantleStation_1".
According to the defined "if-then-else" condition, only one piece of material goes to "DismantleStation_1". This is fictitiously divided into two parts, where one goes through "Overlay" to the "Warehouse_1" warehouse, which guarantees the total number of pieces in the warehouse with the value 500 . The second part in the form of drawing and model goes to "Buffer 3", which is just an auxiliary element connecting "DismantleStation_1" with the "Program_1" window. The material goes to the "Angling" window, where it is processed to the form suitable for clamping to the machining centre. The pieces processed in this way are collected in the "Warehouse_1" warehouse, where the

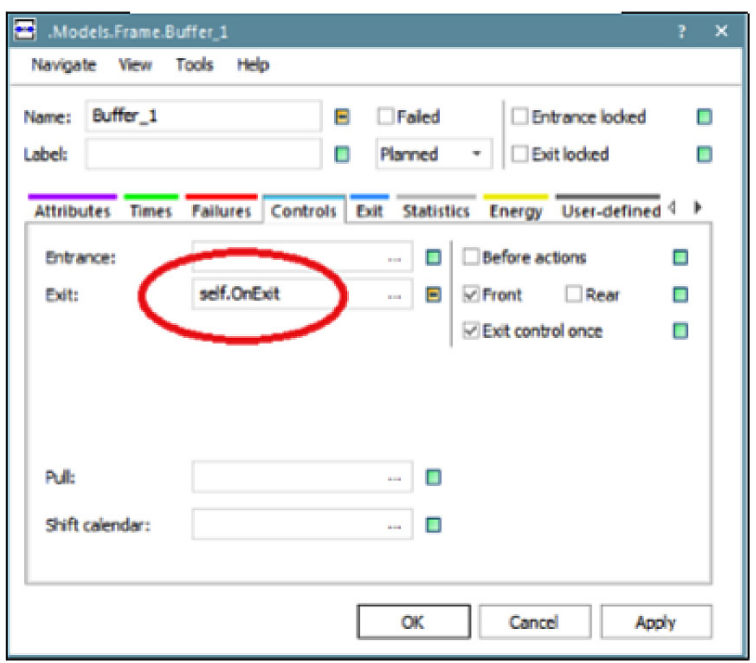

Fig. 6. Card "Controls" of the "Buffer_1" window

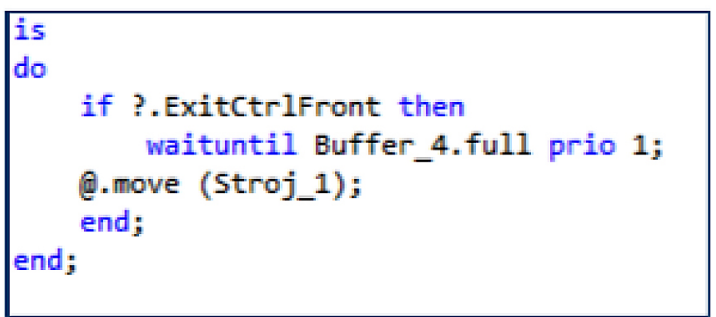

Fig. 7. Condition of the "Warehouse_1" window

is
do Kontrola_1 : = kontrola_1+1;
end;

Fig. 8. Condition of the "Machine_1" window 
"wait until" condition is defined (Figure 7). Its function is to control the input value of the "Buffer_4" window. If its value has the state equal to 1, i.e. the CNC program was created, it sends the material to the "Machine_1" machine, where the first operation is performed.

The "Control_1" condition was set on the input in the first machine (Figure 8). It is used to check the pieces, which passed through the "Machine_1" machine.

The set of elements located behind the first "Machine_1" machine is set for the department of technical control (DTC). The conditions for this system are created by algorithms of control for the produced parts (Figure 9). It contains a counter of pieces. At the same time, they define the conditions that only every fifth produced piece passes through the check and the counter is reset after reaching the value equal to 5 . The others go directly to the "Warehouse_2" warehouse.

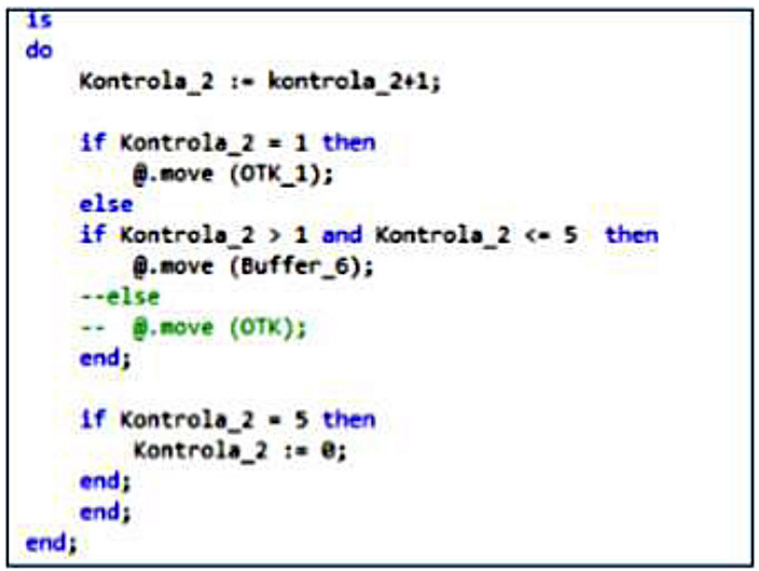

Fig. 9. Condition of the "Buffer_5" window
The production process (Figure 10) presents the first machine clamping from the input information and material to "Machine_1".

Production of the specified part requires six work operations, which can be performed by three machines (for operational reasons). According to the sample of the first clamping, the production continues until the point of the third clamping, when the products are stored and the whole process continues until the first machine on which the first clamping is performed is released. "Warehouse_4" has the condition (at its output) which guarantees the continuation of the production process by

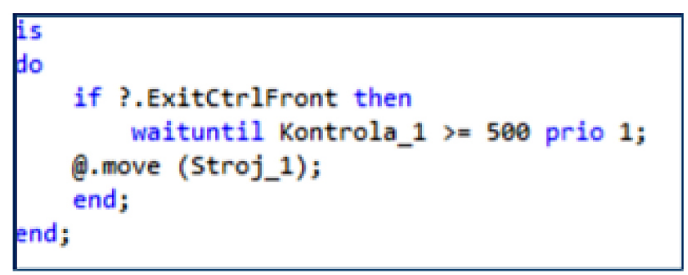

Fig. 11. Condition of the "Warehouse_4" window

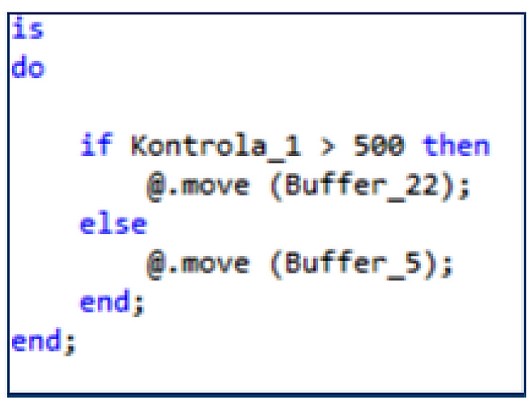

Fig. 12. Condition of the "Machine_1" window

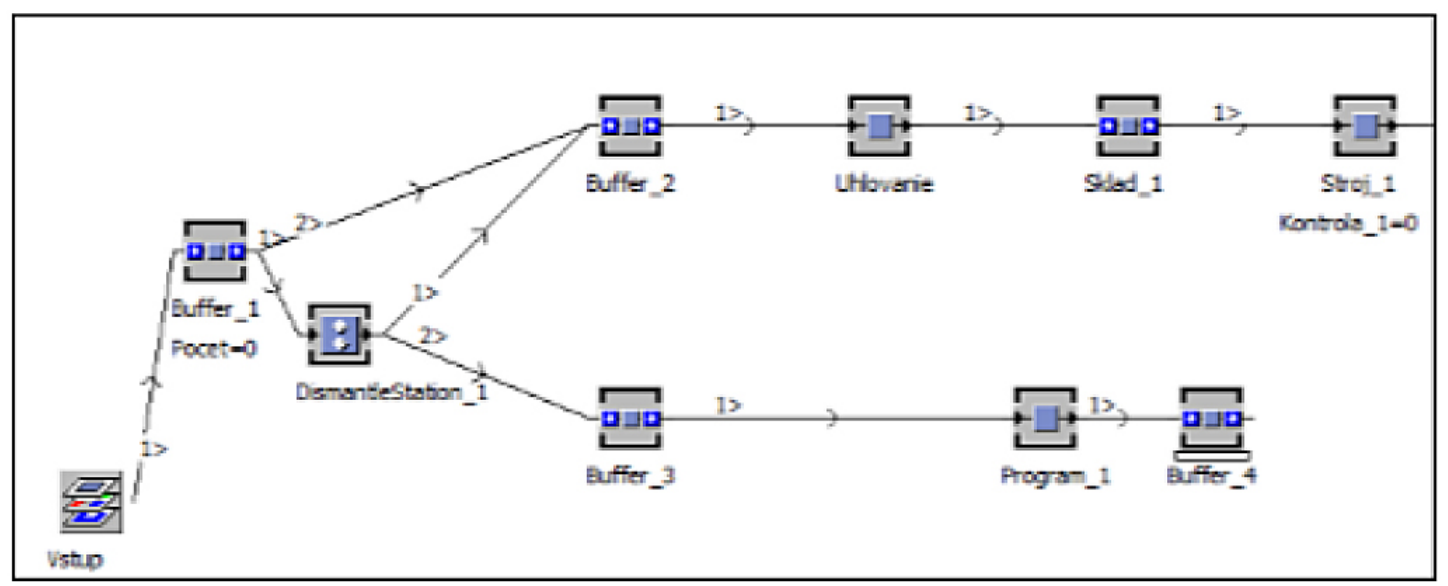

Fig. 10. Part of the program presenting the process from its beginning to the "Machine_1" window 


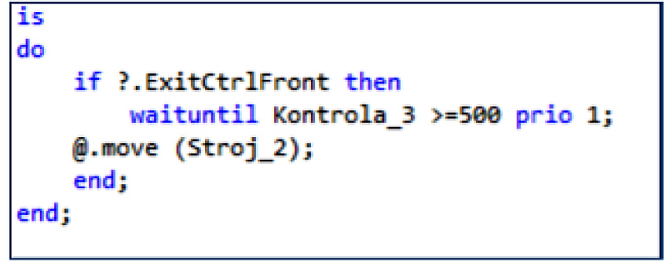

Fig. 13. Condition of the "Warehouse_5" window

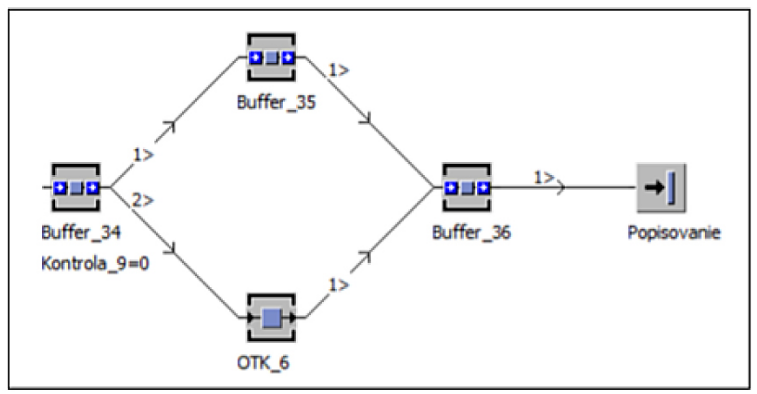

Fig. 14. Ending of the simulation

clamping 4 when 500 pieces pass through the machine number 1 .

The condition of the output "if-then-else" (Figure 12) was set in the first "Machine_1" machine. If "Control_1" is greater than 500, the material can go to the auxiliary "Buffer_22" window, where the production process continues. Otherwise, the pieces go to the "Buffer_5" window.

In the next "Warehouse 5" warehouse, it was set the same condition (Figure 13) on the output as in the previous warehouse. If "Control_3" is greater or equal to 500 , the material can go to the"Machine 2" machine.
The production continuous in a similar way until the 6th clamping. The production process ends using the function "Labeling" (Figure 14), in which the products are described according to the customer's requirements

The output of the simulation presents the presentation of the percentage of machines utilization and the duration of downtimes (Figure 15). The total time of simulation for the production of 500 pieces was 13 days, 23 hours, and 54 minutes.

Machine_1, Machine_2, Machine_3 have different processing time, which causes an unbalanced flow of products within the production process. The process realized by Machine 1 is $15 \%$ longer than the process on Machine_2. Machine 3 has the longest processing time in terms of production complexity compared to the previous machines. As a result, there are interruptions in the production process and the associated utilization of the production capacities of machines. This means that the material processed on Machine 1 is distributed for processing on Machine_2, which the given activity performs at a time when Machine_3 is still occupied by the ongoing process and thus the material has to wait for processing on Machine_3. There are equalizing "Buffers" among machines but they do not have sufficient capacity and therefore the machines are unevenly loaded. The most objectional state is on Machine_2, where it presents up to one-quarter of the available time fund.

From the mentioned outputs it can be stated that there is a significant downtime on Machine_2. As a result of the above said, the procedure of production process optimization was chosen by the help of further simulation for higher efficiency of machines.

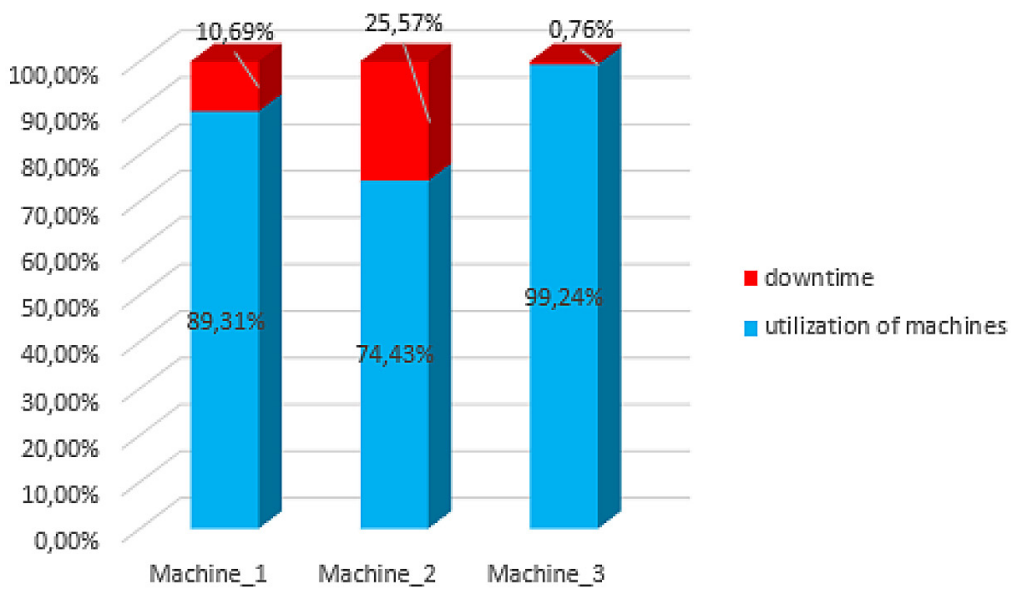

Fig. 15. The output of the simulation 


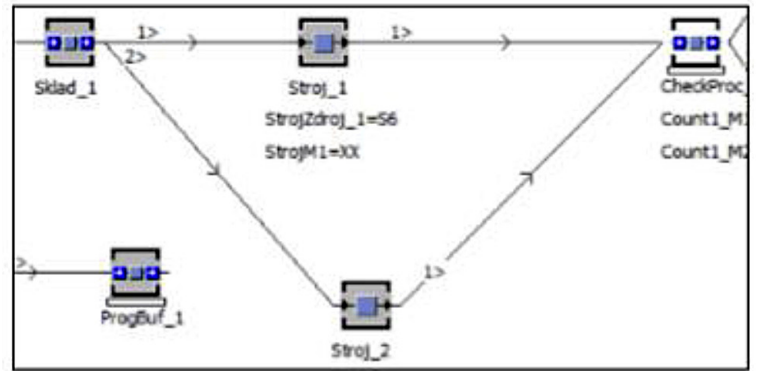

Fig. 16. Illustration of the first clamping

\section{SIMULATION EXPERIMENT - DETERMINATION OF DEVICES USING}

The experimental part of the study optimized the production process used by the evaluated enterprise. Several variants were proposed from the redistribution of devices activities so that the clamping with the longest time in the production process, were processed by several devices at the same time. By this activity, the total time required for the production of parts was shortened, whereas devices utilization was increased and downtime eliminated.

The following proposal of redistribution was considered - clamping No. 1 - divided into Machine_1 and Machine_2, clamping No. 2 - divided into Machine_3 and Machine_1, clamping No. 3 - divided into Machine_2, Machine_3 and Machine_1, clamping No. 4 - divided into Machine_1 and Machine_2, clamping No. 5 - realized by the Machine_3, clamping No. 6 - divided into Machine_1 and Machine_2.

Figure 16 presents the first clamping, which was divided into "Machine_1" and "Machine_2" devices after modifications. This operation ended at both machine centres at the same time. "Machine_3" worked at the second clamping and after the process ends also "Machine_1". "Machine_2" processed the semi-finished material by the third clamping. After the process ending on the other two machines, they were connected to the second working machine centre, i.e. all available devices worked on the third clamping together until they are completed. The fourth clamping was added to the "Machine_1" and "Machine_2" machines. "Machine_3" performed the fifth process of the part production. The final operation was realized by Machines 1 and 2 .

Due to the complexity of the proposed optimization, it was necessary to change the conditions on individual sections of the simulation model.
There are input and output conditions in every machine. For example, the times for clamping are determined at the input, and individual pieces are added to different departments of technical control at the output. For example in the machine room, "Machine_1" is the marking "S1" (Figure 17), this means that the product came from the "Warehouse_1" warehouse to "Machine_1". This machine can automatically identify from which warehouse the material came, and changes the process times accordingly. "Machine_2" and "Machine_3" have the same conditions.

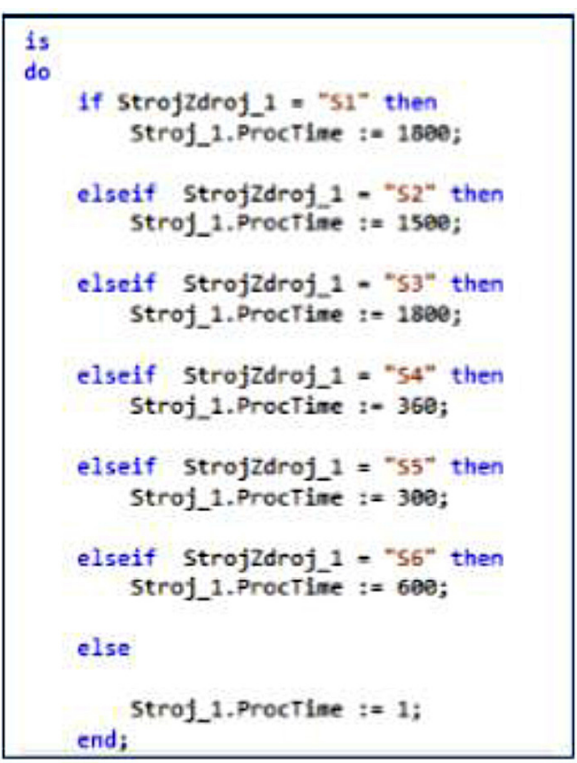

Fig. 17. Condition of the "Machine_1" window on input

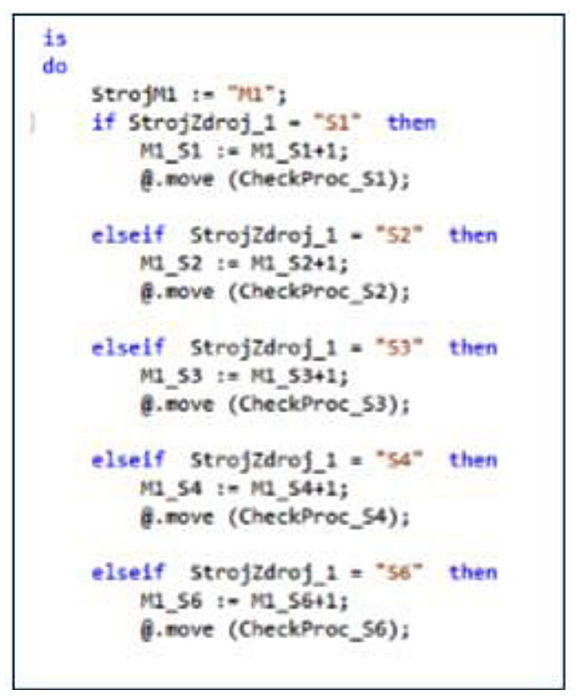

Fig. 18. Condition of the "Machine_1" window on the output 


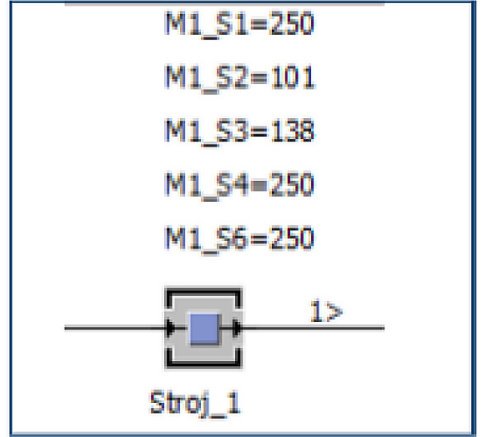

Fig. 19. Auxiliary conditions of the "Machine 1" window

The output of the machine is marked "M1" which means that if the semi-finished product came from "Machine 1" and from the source "S1" which presents "Warehouse_1", the pieces go to "ChekProc_S1" representing the division for the department of technical control (DTC). This condition helps to identify the pieces according to which warehouse they came from.

There are also conditions in the machines (Figure 19), which help to monitor, how many pieces are made in a given machine and where these pieces came from. The "M1_S1" expression expresses that the piece was in the first machine and it came from the first warehouse. Similarly, the "M1_S2" expression represents that the piece from the second warehouse has entered to the first machine.

In the "CheckProc" window, the pieces are divided and must be checked (Figure 20). For example, "CheckProc_S1" has defined that if the material came from "Machine_1", every 5th piece will be moved to the department of technical control "OTK_1 (DTC)". If the material came

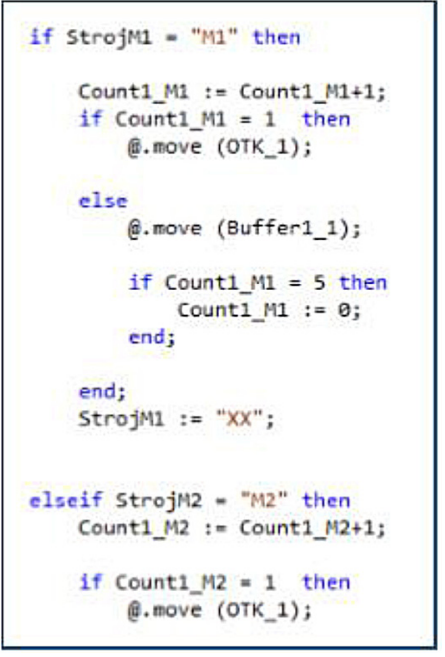

Fig. 20. Condition of the window "CheckProc_S"

from "Machine_2", it must also be checked every 5th piece on "OTK_1 (DTC)".

It was necessary to apply the "Occupied" condition to all warehouses because all machines are still occupied. For example, "Warehouse_4" (Figure 21) must first wait until the "ProgBuf_4" will not be full, and then it starts to be assigned to the machines. First, it determines if "Warehouse 3" or "Machine 2" are still busy. If yes, the pieces start in "Machine_1" and when the value "true" changes to "false", the fourth warehouse can release its pieces to the "Machine_1". All warehouses operate under these conditions and detect if the machines and other warehouses are still busy.

On the basis of the realized simulation experiment, it is possible to state a shortening of the total simulation time in comparison with the real production process in the evaluated enterprise.

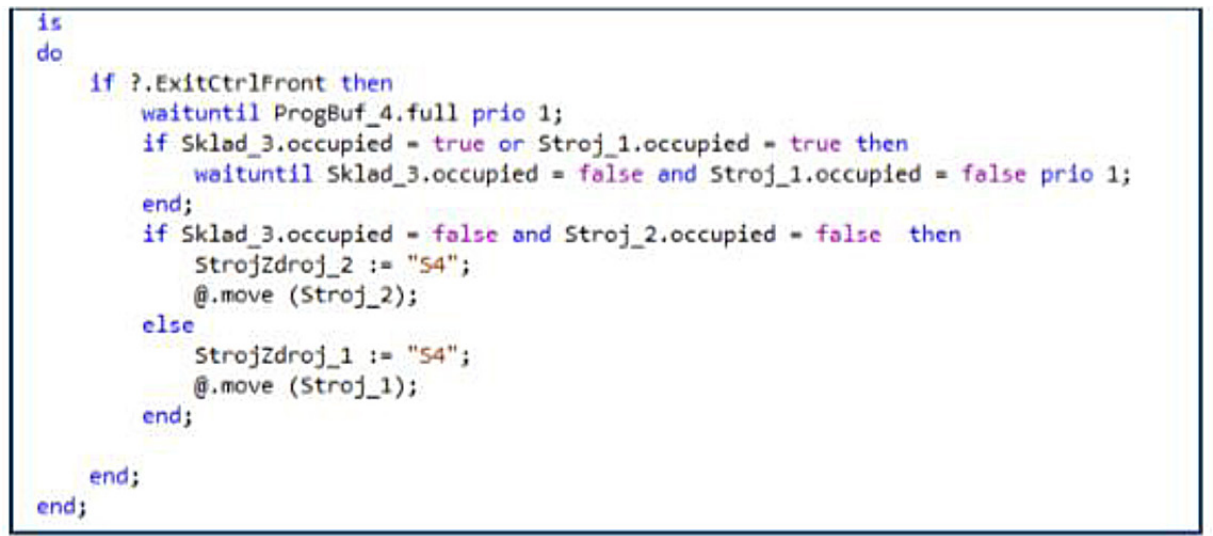

Fig. 21. Condition of the "Warehouse_4" window 


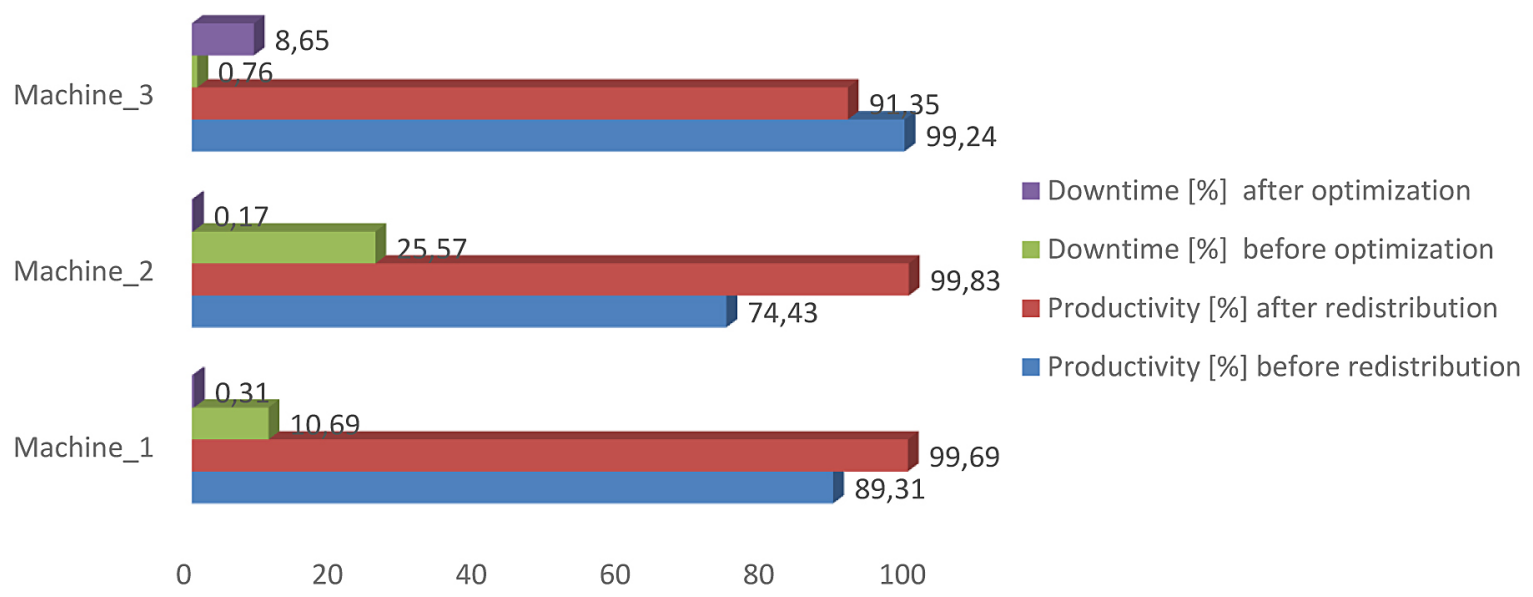

Fig. 22. Results of the simulation/comparison of the simulation results and real results from the enterprise

The optimized production process will enable the production of 500 pieces of parts by 3 machines with a duration of 12 days, 941 minutes. The time saving is 1 day, 493 minutes. Moreover, it is possible to state, decreasing the percentage of downtimes for the production process (Figure 22).

On the basis of the analyzes, which pointed out the imbalance of the material flow within the production process, which caused the uneven utilization of machines, a change of material flow modification was designed. The results of the change presented a improvement in the production process. Primarily, an increase in the use of time capacities of machines was achieved, and the differences among them are negligible and are above $90 \%$. The modifications were the clearest on Machine 2, which was the worst rated in the analysis and achieved an increase of more than $25 \%$. A similar increase was monitored on Machine 1 . In contrast, Machine 3 slightly decreased in terms of time utilization to $91 \%$, but ultimately, it is no problem, because the overall efficiency of the production process was increased and the realized changes were positively reflected in the efficiency of the production process.

\section{CONCLUSION}

Within the paper, the case study was prepared, which, owing to the application of a prescriptive approach, provided the information on the use capacity of devices. Thus, the downtimes of devices were identified and quantified, which reached almost $25 \%$. After the processing of the designs, the verification of the design was realized again using simulation experiments, the result of which brought an increase in capacity and effectiveness of the productivity reaching over $90 \%$. The presented model of this case study is not only a single-use study. Its design and variability make it suitable for regular application in the planning of production capacities and the creation of production plans.

Further research in this field is directed to more broadly virtualization of simulated production processes. The priority is the implementation of the tools of virtual reality. Their use extends the possibility of simulation model and analysis of production processes will become even more detailed. The obtained knowledge can be further used with other devices and technological equipment, enabling the creation of strategic and production plans.

\section{Acknowledgements}

This contribution is the result of the projects VEGA 1/0638/19, KEGA 049TUKE-4/2020, KEGA 012TUKE-4/2019

\section{REFERENCES}

1. Fowler J.W. and Rose O. Grand Challenges in Modeling and Simulation of Complex Manufacturing Systems. Simulation: the Society for Modeling and Simulation International 80, 2004, 469-476.

2. Bucki R. and Chramcov, B. Modelling and simulation of the order realization in the serial production system. International Journal of Mathematical Models and Methods in Applied Sciences 5(7), 2011, 1233-1240.

3. Schindlerova V. and Šajdlerova I. Use of the Dynamic Simulation to Reduce Handling Complexity 
in the Manufacturing Process. Advances in Science and Technology Research Journal 14, 2020, 81-88.

4. Akberdin A.A., Kim A.S. and Sultangaziev R.B. Experiment Planning in the Simulation of Industrial Processes. Steel Transl. 48, 2018, 573-577.

5. Bergmann S. and Strassburger S. Challenges for the Automatic Generation of Simulation Models for Production Systems. Proceedings of Summer Simulation Multiconference - SummerSim '10, 2010, 545-549.

6. Puntel-Schmidt P. and Fay A. Levels of Detail and Appropriate Model Types for Virtual Commissioning in Manufacturing Engineering. IFAC - PapersOnLine 48(1), 2015, 922v927.

7. Savsar M. and Al-Jawini A. Simulation analysis of just-in-time production systems International Journal of Production Economics 42, 1995, 67-78.
8. Chramcov B., Beran P., Daníček L. and Jašek R. A simulation approach to achieving more efficient production systems. International Journal of Mathematics and Computers in Simulation 5, 2011, 299-309.

9. Bakoa B. and Božek P. Trends in Simulation and Planning of Manufacturing Companies Procedia Engineering 149, 2016, 571-575.

10. Lim L.L., Alpan G. and Penz B. A simulation-optimization approach for sales and operations planning in build-to-order industries with distant sourcing: Focus on the automotive industry. Computers \& Industrial Engineering 112, 2017, 469-482.

11. Fabianova J., Kacmary P. and Janekova J. Operative production planning utilising quantitative forecasting and Monte Carlo simulations. Open Eng. 9, 2019, 613-622. 